\title{
CDISC SDTM Unit for Vital Sign Result Terminology
}

National Cancer Institute

\section{Source}

National Cancer Institute. CDISC SDTM Unit for Vital Sign Result Terminology. NCI

Thesaurus. Code C66770.

Terminology codelist used with Unit for Vital Sign Result within the Clinical Data

Interchange Standards Consortium Study Data Tabulation Model. 